\title{
Self-assembly and charge transport properties of a benzobisthiazole end-capped with dihexyl thienothiophene units $\dagger$
}

\author{
Greg J. McEntee, ${ }^{a}$ Filipe Vilela, ${ }^{a}$ Peter J. Skabara, ${ }^{* a}$ Thomas D. Anthopoulos, ${ }^{b}$ John G. Labram, ${ }^{b}$ \\ Steve Tierney, ${ }^{c}$ Ross W. Harrington ${ }^{d}$ and William Clegg ${ }^{d}$
}

\author{
Received 10th August 2010, Accepted 15th October 2010 \\ DOI: 10.1039/c0jm02607g
}

\begin{abstract}
The synthesis of a new conjugated material is reported; BDHTT-BBT features a central electrondeficient benzobisthiazole capped with two 3,6-dihexyl-thieno[3,2-b]thiophenes. Cyclic voltammetry was used to determine the HOMO $(-5.7 \mathrm{eV})$ and LUMO $(-2.9 \mathrm{eV})$ levels. The solid-state properties of the compound were investigated by X-ray diffraction on single-crystal and thin-film samples. OFETs were constructed with vacuum deposited films of BDHTT-BBT. The films displayed phase transitions over a range of temperatures and the morphology of the films affected the charge transport properties of the films. The maximum hole mobility observed from bottom-contact, top-gate devices was $3 \times 10^{-3}$ $\mathrm{cm}^{2} \mathrm{~V}^{-1} \mathrm{~s}^{-1}$, with an on/off ratio of $10^{4}-10^{5}$ and a threshold voltage of $-42 \mathrm{~V}$. The morphological and self-assembly characteristics versus electronic properties are discussed for future improvement of OFET devices.
\end{abstract}

\section{Introduction}

In the design of new materials for organic semiconductor devices, the incorporation of fused thiophenes and other aromatic structures into conjugated architectures provides several advantages over simple non-fused analogues. ${ }^{1-7}$ Increased planarity and rigidity in such structures lead to better $\pi$-orbital overlap and a lower HOMO-LUMO gap, whilst the two-dimensional (i.e. flat) nature of the materials leads to efficient $\pi-\pi$ stacking between molecules. Photoluminescence quantum yields are also known to be higher for fused systems compared to analogous non-fused structures, due to a restriction in torsional freedom. ${ }^{8}$

Compounds consisting solely of core fused aromatics (e.g. pentacene, ${ }^{9,10}$ and dinaphtho-[2,3-b:2', $\left.3^{\prime}-f\right]$ thieno[3,2- $\left.b\right]$ thiophene, DNTT ${ }^{11,12}$ ) represent efficient and comprehensively studied charge transport materials, but they suffer from poor solubility and need to be processed by vacuum deposition techniques to afford thin films. Solution processability is desirable for the cheap manufacture of organic semiconductor devices, so the functionalisation of fused compounds with long-chain or bulky alkyl groups is an obvious means to this end. ${ }^{13}$ The correct choice of substituent, however, can deliver a secondary benefit to the material by utilising van der Waals interactions to complement the self-assembling $\pi-\pi$ stacking motif. Liquid crystalline properties are often observed for such materials and this can be used to good effect in optimising charge transport properties through thermal annealing procedures. ${ }^{14-16}$

${ }^{a}$ WestCHEM, Department of Pure and Applied Chemistry, University of Strathclyde, Glasgow, G1 1XL,UK.E-mail: peter.skabara@strath.ac.uk ${ }^{b}$ Department of Physics and Centre for Plastic Electronics, Blackett Laboratory, Imperial College London, London, SW7 2BW, UK

${ }^{c} M E R C K$ Chemicals, Southampton, SO16 7QD, UK

${ }^{d}$ School of Chemistry, Newcastle University, Newcastle upon Tyne, NE1 $7 R U, U K$

$\dagger$ CCDC reference number 788178. For crystallographic data in CIF or other electronic format see DOI: $10.1039 / \mathrm{c} 0 \mathrm{jm} 02607 \mathrm{~g}$
The introduction of heterocyclic units into fused structures can lead to stabilised HOMOs and wider HOMO-LUMO gaps compared to oligoacenes. ${ }^{17}$ This strategy avoids trap states ${ }^{18}$ that are often seen with pentacene and related materials, leading to instability in air. There has been interest lately in conjugated materials incorporating electron-deficient heterocycles, such as $[1,3,4]$ thiadiazoles ${ }^{19-23}$ and dibromobenzo[1,2- $\left.d ; 4,5-d^{\prime}\right]$ bisthiazole, ${ }^{24-27}$ particularly due to their relevance to n-type materials. ${ }^{28}$ Recently, we reported a novel material (1) based on a benzobisthiazole core with hexylthiophene end units. ${ }^{29}$ This compound self-assembles into a rare example of an organic semiconductor molecule in which there is a three-dimensional arrangement of overlapping orbitals in the bulk: two directions through $\pi-\pi$ stacking and the third through infinite chains of $\mathrm{S} \cdots \mathrm{N}$ close contacts. In an attempt to improve the charge transport properties of this material, we have synthesised an extended analogue of compound $\mathbf{1}$ featuring fused dialkyl thienothiophene end units (BDHTT-BBT). Although the same three-dimensional ordering is not observed in this compound, the material demonstrates a high propensity to self-assemble in the solid state with phase transitions over a short temperature range, which highlights the need to perform studies such as thermal

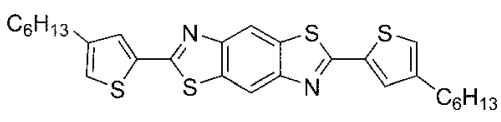

1

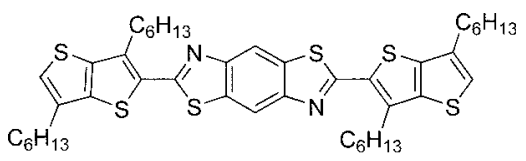

BDHTT-BBT 
annealing for the optimisation of device performance. In this article, we focus on the morphology of thin films of BDHTTBBT annealed at different temperatures and compare these results with the crystal structure of the compound. Organic field effect transistors were fabricated and the hole mobilities of the treated films are discussed.

\section{Results and discussion}

\section{Synthesis}

The synthesis of BDHTT-BBT is presented in Scheme 1. 3,6Dihexyl-thieno[3,2-b]thiophene ${ }^{30}$ (2) was prepared according to the generalised method for dialkyl thienothiophenes, developed by McCulloch et al. ${ }^{16}$ Lithiation of compound 2 by $n$-butyllithium at $-30{ }^{\circ} \mathrm{C}$ in $\mathrm{THF}$ was followed by the addition of trimethyltin chloride, affording compound 3 in $70 \%$ crude yield. After aqueous work-up, the stannyl reagent was used without further purification in the next stage of the synthesis. Microwaveassisted coupling of 2,6-dibromobenzo[1,2- $\left.d ; 4,5-d^{\prime}\right]$ bisthiazole $(4)^{29}$ with a small excess of 3 under Stille conditions gave the target compound BDHTT-BBT in $62 \%$ yield.

\section{Determination of energy levels}

Cyclic voltammetry experiments were performed with $\mathrm{CH}_{2} \mathrm{Cl}_{2}$ solutions of the substrate in $0.1 \mathrm{mM}$ concentration. $\mathrm{TBAPF}_{6}$ was used as the supporting electrolyte in $0.1 \mathrm{M}$ concentration. The working electrode was glassy carbon and the data are referenced against ferrocene, which was used as the internal standard. The voltammogram in Fig. 1 shows a quasi-reversible oxidation peak at $E_{1 / 2 \mathrm{ox}}=+1.05 \mathrm{~V}$ and a reversible reduction peak at $E_{1 / 2 \text { red }}=-1.91 \mathrm{~V}$. A second irreversible oxidation process is seen at $+1.18 \mathrm{~V}$. The corresponding HOMO and LUMO levels for BDHTT-BBT (the onsets of the first oxidation and reduction peaks) are -5.74 and $-2.89 \mathrm{eV}$, respectively. This corresponds to an electrochemical HOMO-LUMO gap of $2.85 \mathrm{eV}$. The HOMO level is quite low-lying compared to that of gold and this presents a problem for charge injection in devices (vide infra). We attempted the electrochemical polymerisation of BDHTT-BBT by repetitive cycling over both of the material's oxidation waves, but no material was detected at the working electrode.
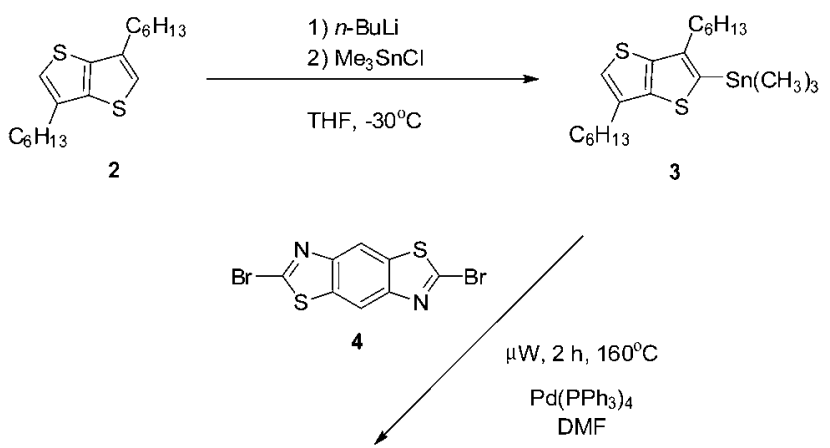

BDHTT-BBT

Scheme 1 Synthetic procedure for the preparation of compound BDHTT-BBT.

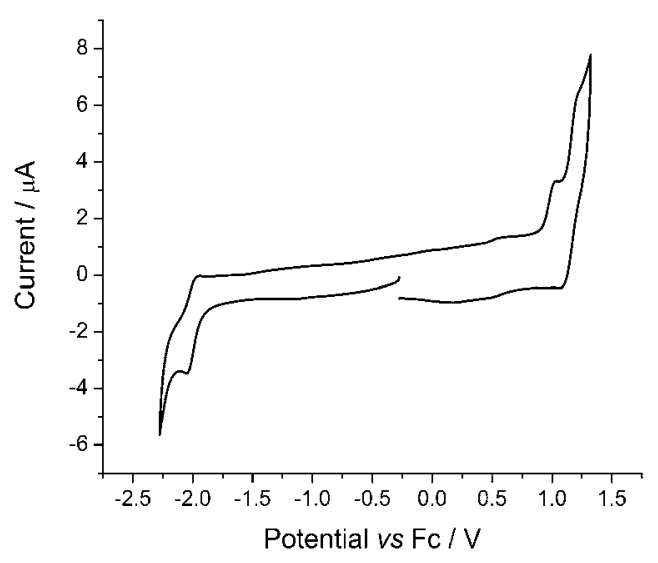

Fig. 1 Cyclic voltammogram of BDHTT-BBT $(0.1 \mathrm{mM})$ in dichloromethane, using a glassy carbon working electrode, silver wire pseudo reference, $\mathrm{Pt}$ wire counter electrode, $0.1 \mathrm{M} \mathrm{TBAPF}_{6}$ electrolyte, scan rate $100 \mathrm{mV} \mathrm{s}^{-1}$.

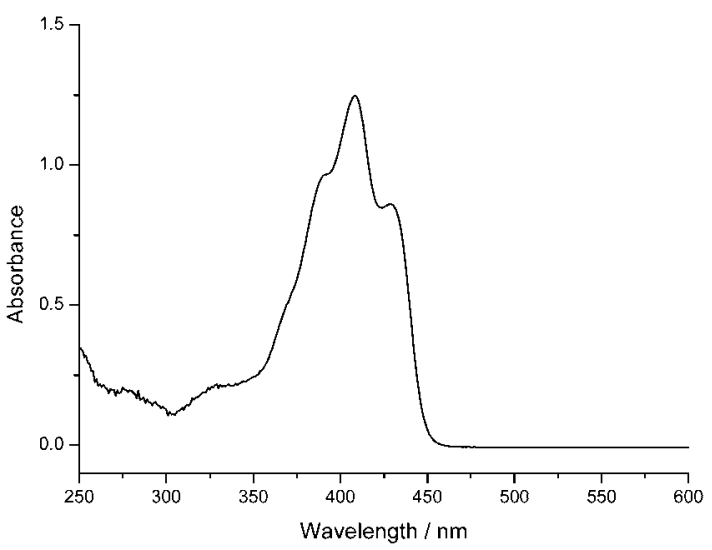

Fig. 2 Absorption spectrum of BDHTT-BBT in dichloromethane solution.

The UV-vis absorption spectrum of BDHTT-BBT was recorded in dichloromethane and can be seen in Fig. 2. The main absorption peak for the material was at $409 \mathrm{~nm}\left(94000 \mathrm{M}^{-1}\right.$ $\left.\mathrm{cm}^{-1}\right)$, with shoulders at $388\left(72500 \mathrm{M}^{-1} \mathrm{~cm}^{-1}\right)$ and $430 \mathrm{~nm}$ $\left(65000 \mathrm{M}^{-1} \mathrm{~cm}^{-1}\right)$. The absorption spectrum for compound $\mathbf{1}$ in chloroform solution displays fine structure (359, 378 and $398 \mathrm{~nm})$ also indicating a rigid conformer in solution state. ${ }^{29}$ The optical HOMO-LUMO gap was calculated to be $2.73 \mathrm{eV}$, which is in close agreement with the HOMO-LUMO gap determined by cyclic voltammetry.

\section{Crystallography}

Long yellow needles of BDHTT-BBT were grown by layered diffusion from acetone-chloroform and the molecular structure of the compound is shown in Fig. 3. The heterocyclic units linked by single bonds show a high degree of coplanarity with maximum dihedral angles of $3.36^{\circ}$ between the central benzobisthiazole core and the dithienothiophene rings. Non-covalent $\mathrm{S} \cdots \mathrm{N}$ interactions between these units act to hold the molecule in a planar conformation, ${ }^{20}$ which should enhance conjugation within the molecule and help to improve charge transport 


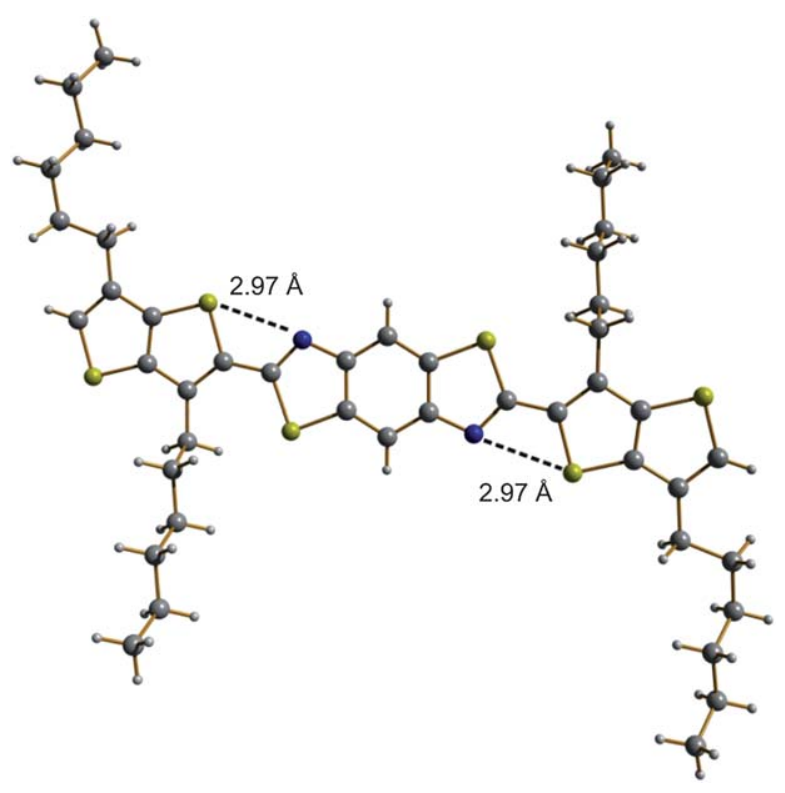

Fig. 3 Molecular structure of BDHTT-BBT.

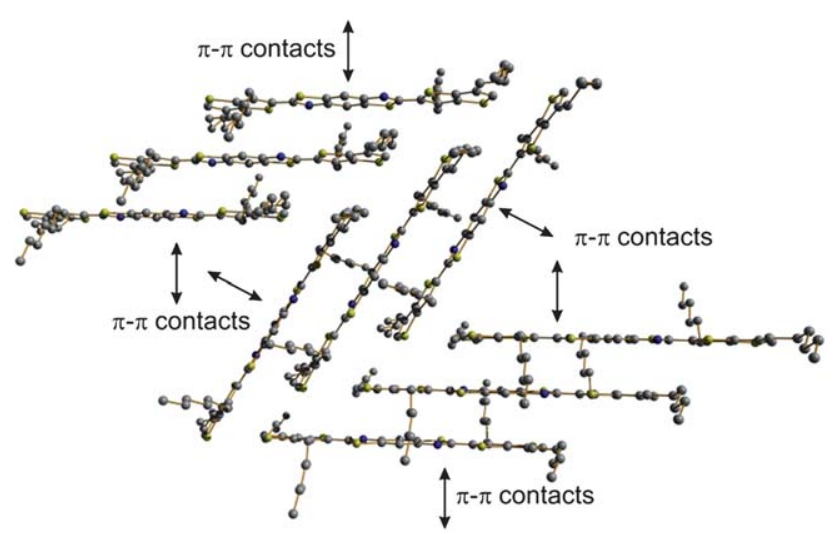

Fig. 4 Packing of BDHTT-BBT to form a herringbone type structure.

through efficient $\pi-\pi$ interactions. The distance between the thienothiophene sulfur and the benzobisthiazole nitrogen atoms is $2.97 \AA$. This is significantly shorter than the sum of the van der Waals radii of the two atoms $(\mathrm{S}+\mathrm{N}=3.35 \AA) .{ }^{31}$

From single-crystal X-ray diffraction of the bulk material, it is seen that the molecules assemble into columns with a slippedstack structure, allowing two stacks to form a herringbone pattern (Fig. 4). The interplanar $\pi-\pi$ distance between molecules is $3.52 \AA$. Between the stacks the molecules' alkyl chains are interdigitated, restricting the $\pi-\pi$ contacts to two dimensions. Also, the intermolecular $\mathrm{S} \cdots \mathrm{N}$ interactions present in the bulk of compound $\mathbf{1}$ are not observed in the packing of BDHTT-BBT, leading to the loss in $3 \mathrm{D}$ ordering. ${ }^{29}$

\section{Thin-film studies}

X-Ray diffraction studies were performed on thin-film samples of BDHTT-BBT drop-cast from chlorobenzene and then annealed at $80-100{ }^{\circ} \mathrm{C}$ (Fig. 5). The results showed that the material has crystalline order in the thin-film state before and after annealing. The initial pre-annealed film shows four main peaks at $d=16.8 \AA, 15.6 \AA, 14.7 \AA$ and $6.9 \AA$ (Fig. 5b). After heating the film at $80{ }^{\circ} \mathrm{C}$ for 16 hours and then $100{ }^{\circ} \mathrm{C}$ for a further two hours, the XRD pattern changed to represent a more uniform film with a single interlayer distance ( $d$-spacing) and a series of higher order diffractions (12.7 $\mathrm{\AA}, 6.4 \AA$, $4.4 \AA$, $3.3 \AA$ and $2.7 \AA$; Fig. 5c). Both films give XRD patterns that are quite different from the set of data obtained from crushed crystals of BDHTT-BBT (Fig. 5a), and therefore a meaningful

(a)

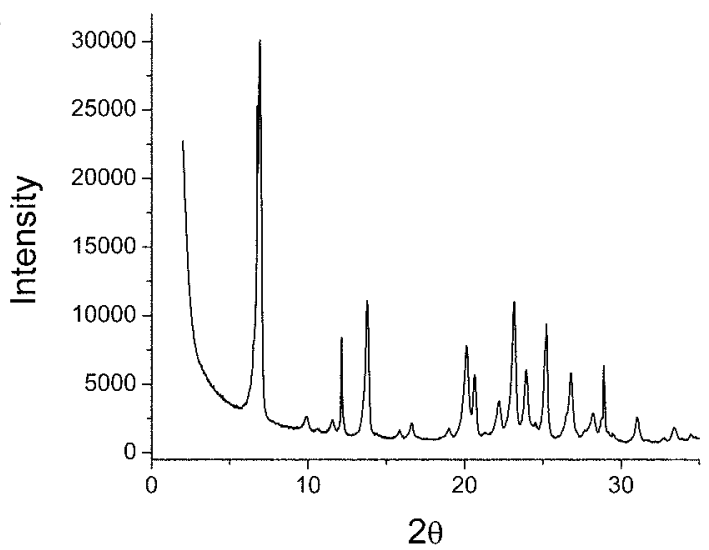

(b)

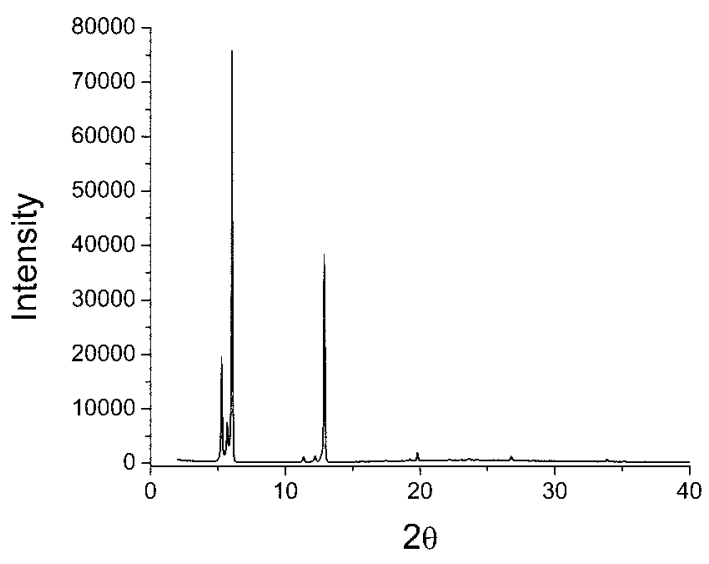

(c)

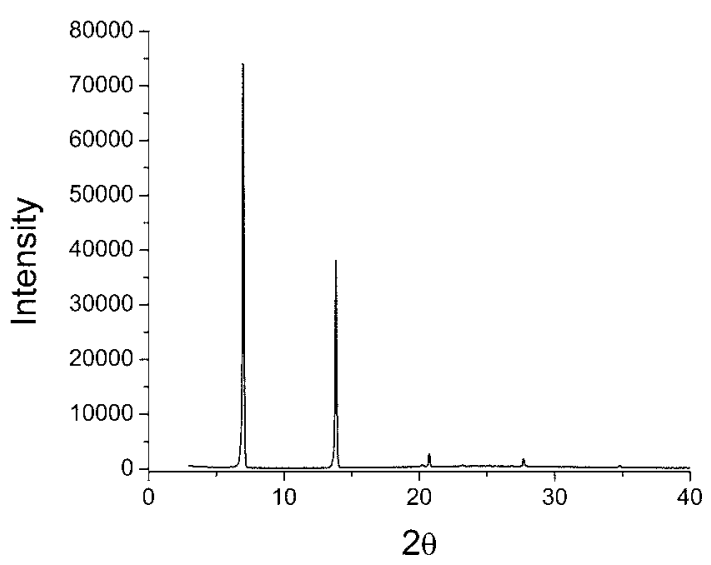

Fig. 5 X-Ray diffraction studies on BDHTT-BBT. (a) Obtained from crushed crystals of BDHTT-BBT; (b) obtained from a thin-film, dropcast from a chlorobenzene solution, then (c) after annealing at $80^{\circ} \mathrm{C}$ for $16 \mathrm{~h}$ and $100^{\circ} \mathrm{C}$ for $2 \mathrm{~h}$. 


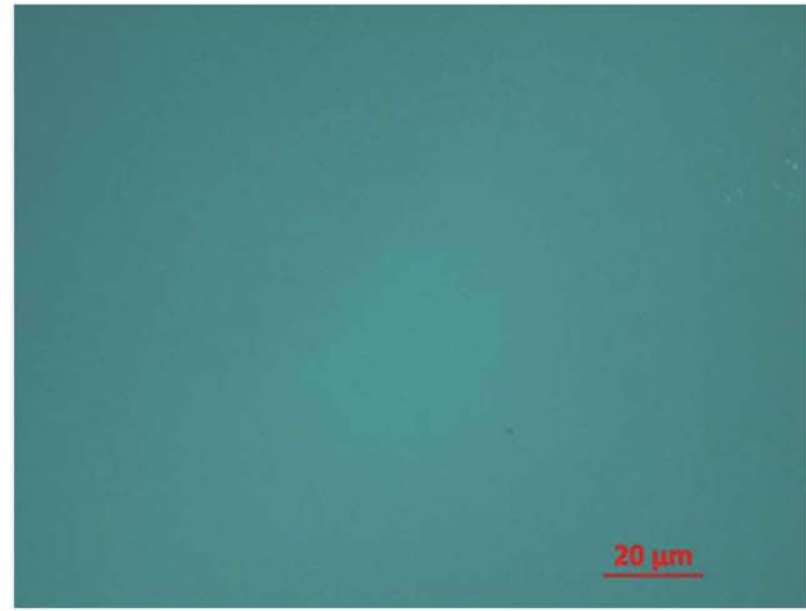

(a)

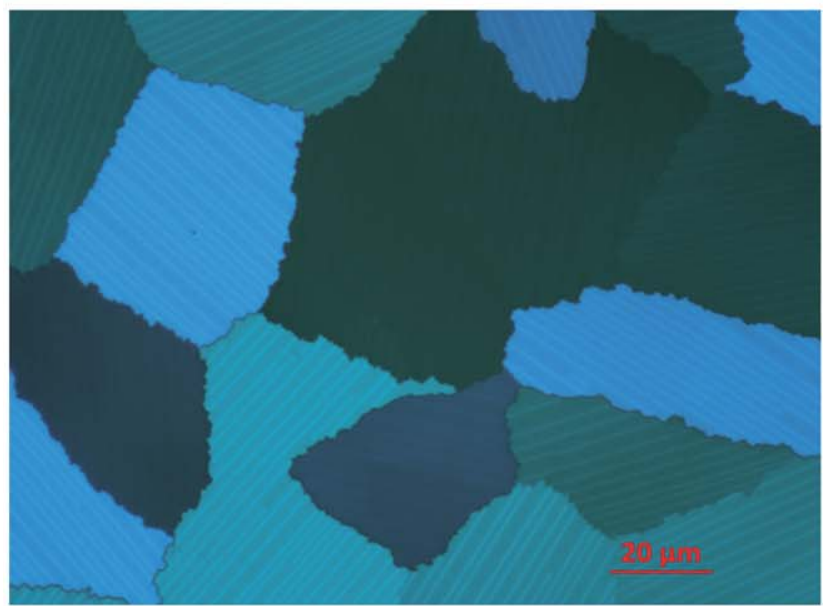

(b)

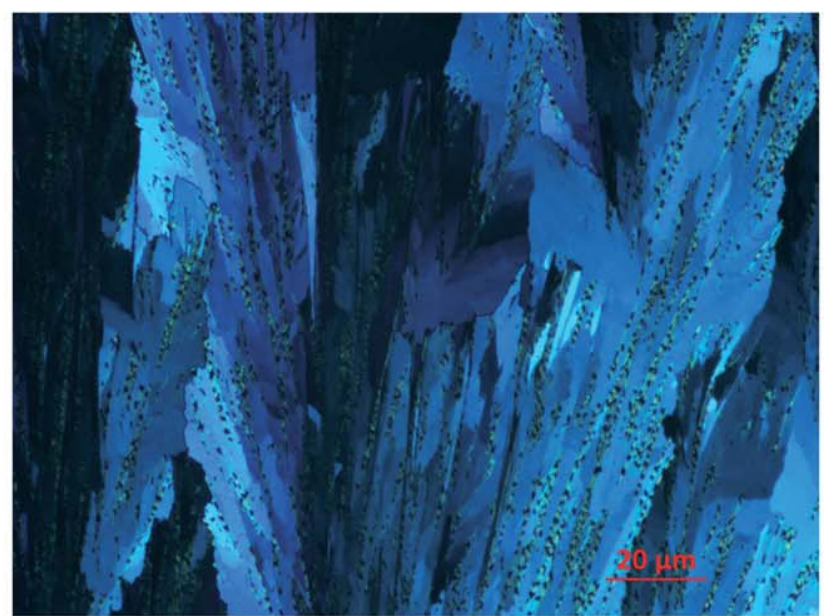

(c)

Fig. 6 Polarised optical microscopy of vacuum deposited BDHTTBBT: (a) as-deposited; (b) crystal $\mathrm{B}$ phase after annealing at $80{ }^{\circ} \mathrm{C}$ for $16 \mathrm{~h}$; (c) crystal E phase after annealing at $80^{\circ} \mathrm{C}$ for $16 \mathrm{~h}$.

comparison with the single-crystal X-ray structure cannot be made.

BDHTT-BBT films were prepared by vacuum deposition. From polarised optical microscopy, the as-deposited film

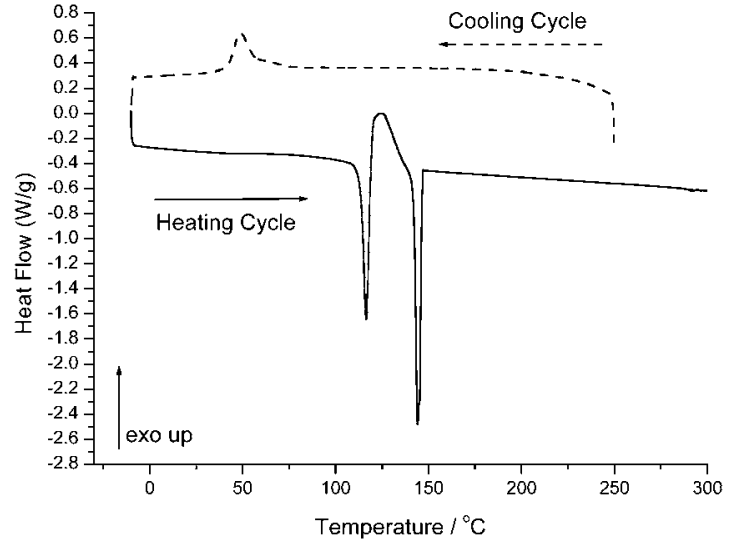

Fig. 7 DSC plot for compound BDHTT-BBT

(Fig. 6a), did not feature any crystalline regions, but after annealing at $80^{\circ} \mathrm{C}$ for 16 hours two distinct phases were observed in the film. The first of these (Fig. 6b), is a crystal B phase, a soft crystal lamellar structure with long-range hexagonal positional order and local rotational disorder. Fig. 6c shows the crystal E phase, which has long-range orthorhombic ordering with local molecular flipping. Raising the annealing temperature to $100{ }^{\circ} \mathrm{C}$ for 2 hours caused the crystal B phase to be replaced entirely by the crystal E phase.

Differential scanning calorimetry (DSC) was performed on a bulk sample of BDHTT-BBT (Fig. 7). After the removal of the thermal history with a heating cycle, it was observed that on heating $\left(-10{ }^{\circ} \mathrm{C}\right.$ to $\left.250{ }^{\circ} \mathrm{C}, 10{ }^{\circ} \mathrm{C} \mathrm{min}^{-1}\right)$ BDHTT-BBT gave a melting point at $145.2^{\circ} \mathrm{C}$ and on cooling a crystallisation point at $48{ }^{\circ} \mathrm{C}$. Subsequent heating displayed two endothermic processes upon heating, at $116.6^{\circ} \mathrm{C}$ and $144.5^{\circ} \mathrm{C}$. The first was assigned to a crystalline polymorph transition.

\section{OFET fabrication and characterisation}

In order to determine the charge transport properties of this material, organic field-effect transistors (OFETs) were fabricated and characterised. Here a bottom-contact, top-gate (BCTG) device architecture was employed (see inset of Fig. 8). This architecture is known to result in good charge-transport characteristics because of a large electrode-semiconductor interfacial $\mathrm{area}^{32}$ and low concentration of trap states at the semiconductordielectric interface. ${ }^{33}$

Fig. 8 shows the transfer characteristics (drain current $I_{\mathrm{D}}$, as a function of gate voltage $V_{\mathrm{G}}$ ) of a BCTG OFET fabricated from BDHTT-BBT. This device had a channel width $(W)$ and length (L) of $500 \mu \mathrm{m}$ and $10 \mu \mathrm{m}$, respectively. Using standard semiconductor models, ${ }^{32}$ the field effect mobility can be determined from eqn (1) and the transfer characteristics of Fig. 8 to be approximately $3 \times 10^{-3} \mathrm{~cm}^{2} \mathrm{~V}^{-1} \mathrm{~s}^{-1}$.

$$
\mu=\frac{2 L}{W C_{i}}\left(\frac{\mathrm{d} \sqrt{I_{\mathrm{D}}}}{\mathrm{d} V_{\mathrm{G}}}\right)^{2}
$$

Here $C_{i}$ is the geometrical capacitance of the gate dielectric $\left(2 \mathrm{nF} \mathrm{cm}{ }^{-2}\right)$. From plots of $I_{\mathrm{D}}{ }^{1 / 2}$ against $V_{\mathrm{G}}$ when biased in the saturation regime the threshold voltage was determined to be 


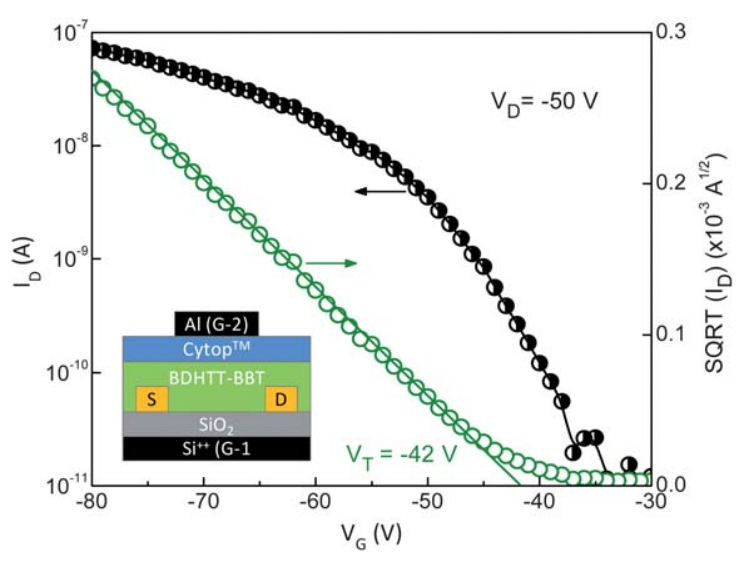

Fig. 8 Transfer characteristics of BDHTT-BBT-based bottom-contact, top-gate organic field-effect transistor $(L=10 \mu \mathrm{m}$ and $W=500 \mu \mathrm{m})$ measured after annealing at $70{ }^{\circ} \mathrm{C}$ for 15 hours under ambient pressure $\mathrm{N}_{2}$. Inset shows the schematic cross-section of the bottom-contact, topgate transistor structure employed here.

approximately $-42 \mathrm{~V}$. The current on-off ratio was estimated to be in the range $10^{4}-10^{5}$. The relatively low mobility and large negative threshold voltage of these transistors are likely to be due to the relatively deep HOMO level of this material, and hence the large injection barrier from the gold source and drain electrodes (work function $\sim-4.8 \mathrm{eV}$ ). Use of lower work function electrodes (such as platinum for example) is expected to improve the performance of these devices significantly. A similar effect could in principle be achieved through the use of suitable work function modifying self-assembling monolayers. ${ }^{34}$ Transistors fabricated from BDHTT-BBT annealed at higher temperatures (i.e. containing the E phase), gave lower field effect mobilities than the device described above.

\section{Experimental}

\section{General}

Melting points were taken using Stuart Scientific apparatus and are uncorrected. ${ }^{1} \mathrm{H}$ NMR spectra were recorded on a Bruker Avance DRX500 instrument at $500 \mathrm{MHz}$ and ${ }^{13} \mathrm{C} \mathrm{NMR}$ at $125 \mathrm{MHz}$; chemical shifts $\left(\delta_{\mathrm{H}}\right)$ are given in parts per million (ppm). Peak multiplicities are denoted by s (singlet), d (doublet), $\mathrm{t}$ (triplet) and $\mathrm{m}$ (multiplet) or by a combination of these: $\mathrm{dd}$ (doublet of doublets), dt (doublet of triplets) and td (triplet of doublets) with coupling constants $(J)$ given in Hertz $(\mathrm{Hz})$. Elemental analyses were conducted on a Perkin Elmer 2400. MALDI-TOF spectra were run on a Shimadzu Axima-CFR spectrometer (mass range 1-150000 Da). X-Ray powder diffraction data were collected using a Siemens D500 diffractometer operating in Bragg Brentano geometry using $\mathrm{Cu} \mathrm{K} \alpha$ radiation. DSC was measured on a TA Instruments DSC Q1000, with all the data being processed with TA Instruments Universal Analysis 200 software. The sample ( $c a$. 2-4 mg), was weighed into a crimped aluminium pan and the analysis was performed under a flow of nitrogen $\left(20 \mathrm{ml} \mathrm{min} \mathrm{min}^{-1}\right)$ through the following steps: equilibrate at $25.0{ }^{\circ} \mathrm{C}$; isothermal for $1.00 \mathrm{~min}$; ramp $10.0{ }^{\circ} \mathrm{C} \mathrm{min}^{-1}$ to $250.0^{\circ} \mathrm{C}$; end of cycle 1 ; isothermal for $1.0 \mathrm{~min}$; ramp $10.0{ }^{\circ} \mathrm{C} \min ^{-1}$ to $-10{ }^{\circ} \mathrm{C}$; end of cycle 2 ; isothermal for $1.00 \mathrm{~min}$; ramp $10.0{ }^{\circ} \mathrm{C} / \mathrm{min}$ to $300.0{ }^{\circ} \mathrm{C}$; end of cycle 3 .

Column chromatography was performed with commercially available solvents and using VWR silica gel $(40-63 \mu \mathrm{m})$. Thin layer chromatography (TLC) was performed using aluminium plates pre-coated with Merck silica gel $60\left(\mathrm{~F}_{254}\right)$ and visualised by ultra-violet radiation and/or iodine vapour. The chemicals were purchased from Sigma-Aldrich and used without further purification, unless stated otherwise. Anhydrous solvents were obtained from a PureSolv solvent purification system.

Cyclic voltammetry measurements were performed on a $\mathrm{CH}$ Instruments 660A Electrochemical Workstation with iR compensation, using anhydrous dichloromethane as the solvent. Absorption spectra were recorded on a UNICAM UV 300 instrument in dichloromethane solution.

\section{(3,6-Dihexyl-thieno[3,2-b] thiophen-2-yl)trimethylstannane (3)}

3,6-Dihexyl-thieno[3,2-b]thiophene (2) (580 mg, $1.88 \mathrm{mmol}$ ) was dissolved in THF $(40 \mathrm{ml})$ and cooled to $-30{ }^{\circ} \mathrm{C}$. $n$-Butyllithium (0.84 ml, $1.89 \mathrm{mmol}, 2.25 \mathrm{M}$ solution in hexane) was added dropwise and the reaction was stirred for $1 \mathrm{~h}$. Trimethyltin chloride (1.88 ml, $1.88 \mathrm{mmol}, 1.0 \mathrm{M}$ solution in THF) was added dropwise and the reaction mixture was stirred for $1 \mathrm{~h}$ at $-30^{\circ} \mathrm{C}$, after which it was left stirring overnight at room temperature. The reaction mixture was washed with saturated ammonium chloride solution and water. The aqueous mixture was extracted with diethyl ether $(3 \times 80 \mathrm{ml})$ and dried over $\mathrm{MgSO}_{4}$. The solvent was removed under reduced pressure and the product was obtained as a clear oil (620 mg, 70\%). The material was used without further purification. ${ }^{1} \mathrm{H} \mathrm{NMR}\left(\mathrm{CDCl}_{3}, 500 \mathrm{MHz}, \mathrm{ppm}\right)$ : $\delta 6.94(\mathrm{~s}, 1 \mathrm{H}), 3.76(\mathrm{t}, J=6.5,4 \mathrm{H}), 1.87(\mathrm{~m}, 2 \mathrm{H}), 1.74(\mathrm{~m}, 2 \mathrm{H})$, $1.34(\mathrm{~m}, 12 \mathrm{H}), 0.9(\mathrm{t}, J=7,6 \mathrm{H}), 0.42(\mathrm{~s}, 9 \mathrm{H})$.

\section{2,6-Bis(3,6-dihexyl-thieno[3,2-b]thiophen-2-yl)benzo[1,2-d:4,5- $d^{\prime}$ ]bis(thiazole) (BDHTT-BBT)}

The target compound was prepared by microwave-assisted Stille cross coupling. 2,6-Dibromobenzo[1,2- $\left.d: 4,5-d^{\prime}\right]$ bisthiazole (4) $(0.184 \mathrm{~g}, 0.53 \mathrm{mmol})$ and tetrakis(triphenylphosphine)palla$\operatorname{dium}(0)(61 \mathrm{mg}, 10 \mathrm{~mol} \%)$ were added to a microwave reaction vessel. The vessel was purged with nitrogen before (3,6-dihexylthieno[3,2-b]thiophen-2-yl)trimethylstannane (3) (546 mg, $1.158 \mathrm{mmol})$ in DMF ( $3 \mathrm{ml})$ was added. The reaction mixture was heated to $160{ }^{\circ} \mathrm{C}$ for a period of $2 \mathrm{~h}$. The resultant mixture was poured into chloroform and washed with $2 \mathrm{M} \mathrm{HCl}(2 \times 100 \mathrm{ml})$ and water $(2 \times 100 \mathrm{ml})$. The organic layers were dried over $\mathrm{MgSO}_{4}$ and the solvent was removed under reduced pressure. The crude material was purified by column chromatography (hexane: $\mathrm{CH}_{2} \mathrm{Cl}_{2} 2$ : 1). Recrystallisation by layered diffusion from a chloroform/acetone solution afforded the product as long yellow needle-like crystals (265 mg, 62\% yield). MPt $142-144{ }^{\circ} \mathrm{C}$, ${ }^{1} \mathrm{H} \mathrm{NMR}\left(\mathrm{CDCl}_{3}, 500 \mathrm{MHz}, \mathrm{ppm}\right): \delta 8.48(\mathrm{~s}, 2 \mathrm{H}), 7.11(\mathrm{~s}, 2 \mathrm{H})$, $3.21(\mathrm{t}, J=8,4 \mathrm{H}), 2.76(\mathrm{t}, J=8,4 \mathrm{H}), 1.87(\mathrm{~m}, 4 \mathrm{H}), 1.80(\mathrm{~m}, 4 \mathrm{H})$, $1.55(\mathrm{~m}, 4 \mathrm{H}), 1.38(\mathrm{bm}, 20 \mathrm{H}), 0.93(\mathrm{t}, J=6,12 \mathrm{H}) .{ }^{13} \mathrm{C} \mathrm{NMR}$ $\left(\mathrm{CDCl}_{3}, 125 \mathrm{MHz}, \mathrm{ppm}\right): \delta 161.6,150.6,141.6,140.2,137.6$, 135.7, 134.6, 132.18, 123.98, 114.4, 31.6, 29.8, 29.6, 29.1, 29.0, 28.7, 22.6, 14.1. Anal. Calcd for $\mathrm{C}_{44} \mathrm{H}_{56} \mathrm{~N}_{2} \mathrm{~S}_{6} ; \mathrm{C}, 65.57 ; \mathrm{H}, 7.06$; N, 3.49; S, 23.89; found C, 65.62; H, 7.01; N, 3.48; S, 24.19\%. 
MALDI-TOF (retinoic acid matrix, reflectron mode): $804.15 \mathrm{M}^{+}$.

X-Ray crystallography. Crystal data for $1: \mathrm{C}_{44} \mathrm{H}_{56} \mathrm{~N}_{2} \mathrm{~S}_{6}, M=$ 805.3, monoclinic, space group $P 2{ }_{1} / c, a=13.180(8), b=$ 21.194(13), $c=7.735(5) \AA, \beta=95.299(7)^{\circ}, V=2151(2) \AA^{3}$, $Z=2, T=120 \mathrm{~K}$, synchrotron radiation (Diamond Light Source beamline I19, $\lambda=0.6889 \AA ⿻ 丿$. measured, 3763 unique, $R_{\text {int }}=0.048 ; 235$ refined parameters, $R=0.050\left(F, F^{2}>2 \sigma\right), R_{\mathrm{W}}=0.134$ ( $F^{2}$, all data), goodness-of-fit 1.03, final difference map extremes +1.20 and -0.38 e $\AA^{-3}$ (see ESI†).

OFET fabrication. OFETs were fabricated on highly doped silicon substrates with a thermally grown $400 \mathrm{~nm}$ layer of silicon dioxide. The $\mathrm{SiO}_{2}$ layer was treated with the primer hexamethyldisilazane (HMDS). HMDS has been reported to improve charge transport in OFETs due to improved crystal formation. ${ }^{35}$ $45 \mathrm{~nm}$ gold source and drain electrodes were then thermally evaporated onto the substrates through shadow masks. A $50 \mathrm{~nm}$ layer of BDHTT-BBT was deposited by vacuum deposition at a base pressure of $10^{-9}$ bar and an average rate of $1 \AA^{\circ} \mathrm{s}^{-1}$. The devices were then annealed for 15 hours at $70{ }^{\circ} \mathrm{C}$ to improve the crystal structure and charge-transport properties of the films. A fluorocarbon polymer dielectric (CYTOP ${ }^{\mathrm{TM}}$ ) was deposited on top of the semiconductor by spin-coating. Finally, $80 \mathrm{~nm}$ aluminium gate electrodes were deposited by vacuum deposition. Electrical characterisation was carried out in $\mathrm{N}_{2}$ at atmospheric pressure using a Keithley 4200 semiconductor parameter analyser.

\section{Summary}

We have presented the synthesis and electrochemical and solidstate properties of a new material (BDHTT-BBT). This compound was developed from a shorter-chain analogue, $\mathbf{1}$, to give a higher HOMO level and facilitate charge injection into the organic semiconductor. The HOMO of BDHTT-BBT was found to be $-5.7 \mathrm{eV}$, whereas that of $\mathbf{1}$ is $-6.0 \mathrm{eV} .{ }^{29}$ However, in the bulk, the compound features different thermal behaviour to vacuum deposited films and the latter clearly show liquid crystalline textures. The hole mobilities of films containing the crystal B phase are higher than those of the E phase and this is commensurate with the higher level of disorder inherent in the latter. The mosaic nature of the crystal B phase is due to domains of molecules with the long axes lying in different directions. This type of structure will lead to grain boundaries and will impair charge transport in the bulk, which accounts for the unanticipated drop in mobility for BDHTT-BBT compared to compound 1. These observations are in good agreement with other systems where the degree of order intimately controls charge mobility, and that by being able to tune this, either by changes of deposition conditions (for instance, changing the annealing temperature, the nature of the substrate or film thickness), one can improve the charge mobility within a device. ${ }^{36}$ We have looked at different methods for processing films of BDHTT-BBT and investigated annealing effects. Whilst the photophysics of these films and the corresponding HOMO/ LUMO energy levels will only experience small perturbations, the difference in morphology impacts significantly on the charge transport characteristics of the material.

\section{Acknowledgements}

FV and JGL thank the EPSRC for funding (EP/E02741 and EP/ F056389/1). WC and RWH thank EPSRC for funding the National Crystallography Service and Diamond Light Source for access to synchrotron facilities. The authors also thank Prof. John W Goodby (University of York) for discussions on the LC properties of BDHTT-BBT.

\section{References}

1 P. J. Skabara, chapter in Handbook of Thiophene-Based Materials, ed. I. F. Perepichka and D. F. Perepichka, John Wiley \& Sons Ltd., Chichester, 2009.

2 I. Afonina, P. J. Skabara, F. Vilela, A. L. Kanibolotsky, J. C. Forgie, A. K. Bansal, G. A. Turnbull, I. D. W. Samuel, J. G. Labram, T. D. Anthopoulos, S. J. Coles and M. B. Hursthouse, J. Mater. Chem., 2010, 20, 1112-1116.

3 T. Ohmae, T. Nishinaga, M. Wu and M. Iyoda, J. Am. Chem. Soc., 2010, 132, 1066-1074.

4 P. Gao, D. Cho, X. Y. Yang, V. Enkelmann, M. Baumgarten and K. Mullen, Chem.-Eur. J., 2010, 16, 5119-5128.

5 J. Arago, P. M. Viruela, E. Orti, R. M. Osuna, B. Vercelli, G. Zotti, V. Hernandez, J. T. L. Navarrete, J. T. Henssler, A. J. Matzger, Y. Suzuki and S. Yamaguchi, Chem.-Eur. J., 2010, 16, 5481-5491.

6 S. Shinamura, E. Miyazaki and K. Takimiya, J. Org. Chem., 2010, 75 , $1228-1234$.

7 Y. Liu, C. A. Di, C. Y. Du, Y. Q. Liu, K. Lu, W. F. Qiu and G. Yu, Chem.-Eur. J., 2010, 16, 2231-2239.

8 G. Barbarella, L. Favaretto, G. Sotgiu, L. Antolini, G. Gigli, R. Cingolani and A. Bongini, Chem. Mater., 2001, 13, 4112-4122.

9 D. Braga and G. Horowitz, Adv. Mater., 2009, 21, 1473-1486.

10 A. Facchetti, Mater. Today, 2007, 10, 28-37.

11 U. Zschieschang, F. Ante, T. Yamamoto, K. Takimiya, H. Kuwabara, M. Ikeda, T. Sekitani, T. Someya, K. Kern and H. Klauk, Adv. Mater., 2010, 22, 982-985.

12 T. Yamamoto and K. Takimiya, J. Am. Chem. Soc., 2007, 129, 2224 2225.

13 S. Allard, M. Forster, B. Souharce, H. Thiem and U. Scherf, Angew. Chem., Int. Ed., 2008, 47, 4070-4098.

14 I. McCulloch, M. Heeney, C. Bailey, K. Genevicius, I. Macdonald, M. Shkunov, D. Sparrowe, S. Tierney, R. Wagner, W. M. Zhang, M. L. Chabinyc, R. J. Kline, M. D. McGehee and M. F. Toney, Nat. Mater., 2006, 5, 328-333.

15 D. M. DeLongchamp, R. J. Kline, E. K. Lin, D. A. Fischer, L. J. Richter, L. A. Lucas, M. Heeney, I. McCulloch and J. E. Northrup, Adv. Mater., 2007, 19, 833-837.

16 I. McCulloch, M. Heeney, M. L. Chabinyc, D. DeLongchamp, R. J. Kline, M. Coelle, W. Duffy, D. Fischer, D. Gundlach, B. Hamadani, R. Hamilton, L. Richter, A. Salleo, M. Shkunov, D. Sporrowe, S. Tierney and W. Zhong, Adv. Mater., 2009, 21, 1091-1109.

17 M. He and F. Zhang, J. Org. Chem., 2007, 72, 442-451.

18 J. E. Northrup and M. L. Chabinyc, Phys. Rev. B: Condens. Matter, 2003, 68, 041202.

19 M. C. McCairn, T. Kreouzis and M. L. Turner, J. Mater. Chem., 2010, 20, 1999-2006.

20 H. Pang, P. J. Skabara, D. J. Crouch, W. Duffy, M. Heeney, I. McCulloch, S. J. Coles, P. N. Horton and M. B. Hursthouse, Macromolecules, 2007, 40, 6585-6593.

21 T. Yamamoto, T. Yasuda, Y. Sakai, S. Aramaki and A. Ramaw, Macromol. Rapid Commun., 2005, 26, 1214-1217.

22 T. Yasuda, T. Imase, Y. Nakamura and T. Yamamoto, Macromolecules, 2005, 38, 4687-4697.

23 T. Yasuda, T. Imase, S. Sasaki and T. Yamamoto, Macromolecules, 2005, 38, 1500-1503.

24 J. F. Mike, J. J. Inteman, A. Ellern and M. Jeffries-El, J. Org. Chem., $2010,75,495-497$. 
25 E. Ahmed, F. S. Kim, H. Xin and S. A. Jenekhe, Macromolecules, 2009, 42, 8615-8618.

26 J. G. Kang, H. J. Kim, Y. K. Jeong, M. K. Nah, C. Park, Y. J. Bae, S. W. Lee and I. T. Kim, J. Phys. Chem. B, 2010, 114, 3791-3798.

27 M. Mamada, J. Nishida, S. Tokito and Y. Yamashita, Chem. Lett., 2008, 37, 766-767.

28 Y. Yamashita, Chem. Lett., 2009, 38, 870-875.

29 H. Pang, F. Vilela, P. J. Skabara, J. J. W. McDouall, D. J. Crouch, T. D. Anthopoulos, D. D. C. Bradley, D. M. De Leeuw, P. N. Horton and M. B. Hursthouse, Adv. Mater., 2007, 19, 4438-4442.

30 Y. J. He, W. P. Wu, G. J. Zhao, Y. Q. Liu and Y. F. Li, Macromolecules, 2008, 41, 9760-9766.

31 A. Bondi, J. Phys. Chem., 1964, 68, 441-451.
32 J. Zaumseil and H. Sirringhaus, Chem. Rev., 2007, 107, 1296-1323.

33 R. Hamilton, J. Smith, S. Ogier, M. Heeney, J. E. Anthony, I. McCulloch, J. Veres, D. D. C. Bradley and T. D. Anthopoulos, Adv. Mater., 2009, 21, 1166-1171.

34 J. Smith, R. Hamilton, Y. Qi, A. Kahn, D. D. C. Bradley, M. Heeney, I. McCulloch and T. D. Anthopoulos, Adv. Funct. Mater., 2010, 20, 2330-2337.

35 H. Sirringhaus, P. J. Brown, R. H. Friend, M. M. Nielsen, K. Bechgaard, B. M. W. Langeveld-Voss, A. J. H. Spiering, R. A. J. Janssen, E. W. Meijer, P. Herwig and D. M. de Leeuw, Nature, 1999, 401, 685-688.

36 V. Coropceanu, J. Cornil, D. A. da Silva Filho, Y. Olivier, R. Silbey and J.-L. Brédas, Chem. Rev., 2007, 107, 926-952. 